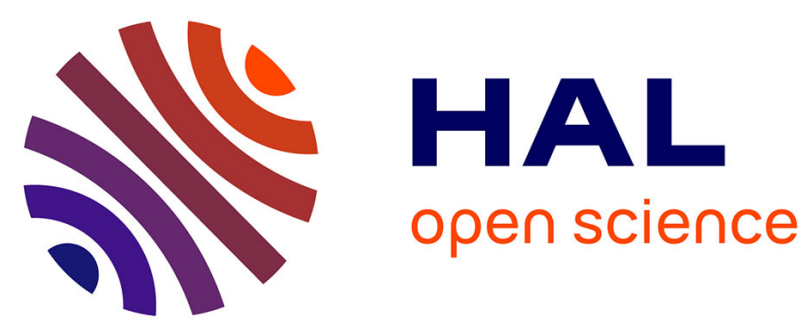

\title{
Synthesis and biological evaluation of oxindoles and benzimidazolines derivatives
}

Samir Messaoudi, Martine Sancelme, Valérie Polard-Housset, Bettina Aboab, Pascale Moreau, Michelle Prudhomme

\section{- To cite this version:}

Samir Messaoudi, Martine Sancelme, Valérie Polard-Housset, Bettina Aboab, Pascale Moreau, et al.. Synthesis and biological evaluation of oxindoles and benzimidazolines derivatives. European Journal of Medicinal Chemistry, 2004, 39, pp.453-458. 10.1016/j.ejmech.2004.01.001 . hal-00136184

\author{
HAL Id: hal-00136184 \\ https://hal.science/hal-00136184
}

Submitted on 12 Mar 2007

HAL is a multi-disciplinary open access archive for the deposit and dissemination of scientific research documents, whether they are published or not. The documents may come from teaching and research institutions in France or abroad, or from public or private research centers.
L'archive ouverte pluridisciplinaire HAL, est destinée au dépôt et à la diffusion de documents scientifiques de niveau recherche, publiés ou non, émanant des établissements d'enseignement et de recherche français ou étrangers, des laboratoires publics ou privés. 


\title{
Synthesis and biological evaluation of oxindoles and benzimidazolinones derivatives
}

\author{
Samir Messaoudi ${ }^{a}$, Martine Sancelme ${ }^{a}$, Valérie Polard-Housset ${ }^{\text {b }}$, Bettina Aboab ${ }^{\text {a }}$, \\ Pascale Moreau $^{\mathrm{a}, *}$, Michelle Prudhomme ${ }^{\mathrm{a}}$ \\ ${ }^{a}$ Laboratoire SEESIB, Université Blaise Pascal, UMR 6504 du CNRS, 24, avenue des Landais, 63177 Aubière cedex, France \\ ${ }^{b}$ Aventis Pharma S. A., oncology department, biochemistry unit, centre de recherche de Paris, 13, quai Jules Guesde, 94400, Vitry sur Seine, France
}

\begin{abstract}
The synthesis of new oxindoles and benzimidazolinones derivatives bearing a sugar residue on the aromatic nitrogen is described. The presence of the glycoside moiety should enhance the solubility of these heterocyclic compounds and/or improve the interaction with the active site of the biological targets. The inhibitory activities of these new compounds toward five kinases were examined: KDR (VEGFR-2), FGFR-1, PDGFR- $\beta$, EGFR and Tie 2. Furthermore, the antibacterial activities of the prepared compounds were tested against two Gram-positive bacteria Bacillus cereus and Streptomyces chartreusis, a Gram-negative bacterium Escherichia coli and a yeast Candida albicans.
\end{abstract}

\section{Introduction}

Growth factors receptors exhibiting a tyrosine kinase activity are a group of transmembrane proteins involved in signal transduction. Their function in many cell types is to drive a wide variety of cellular functions, including growth, differentiation and angiogenesis by transducing growth factor signals from the external environment to intracellular processes. In malignancies, these pathways are often exploited by tumor cells to optimize tumor growth and metastasis. Indeed, alterations in receptor tyrosine kinases pathways have been implicated in oncogenic activation, tumor angiogenesis and mitogenic stimulation [1]. Thus, receptor tyrosine kinases are logical targets for novel anticancer agents development. Among a large number of small molecule receptor tyrosine kinases antagonists, several oxindole derivatives (ATP competitive inhibitors) are in phase I-III clinical development (Fig. 1) [1-4].

In this paper, the synthesis of new oxindoles and benzimidazolinones derivatives bearing a sugar residue on the aromatic nitrogen are described. The presence of the glycoside moiety should enhance the solubility of these heterocyclic

\footnotetext{
* Corresponding author.

E-mail address: pmoreau@chimsrv1.univ-bpclermont.fr (P. Moreau).
}

compounds and/or improve the interaction with the active site of the target enzymes. The inhibitory activities of these new compounds toward five kinases were examined: KDR (VEGFR-2), FGFR-1, PDGFR- $\beta$, EGFR and Tie 2 (receptor tyrosine kinase which is activated by its ligand: angiopoietin 1). Since different kinases activities are involved in the growth of microorganisms and many oxindoles are kinases inhibitors, these new compounds could exert antibacterial activities as it was shown previously for several oxindoles derivatives $[5,6]$. For these reasons, the antibacterial activities of the prepared compounds were tested against two Gram-positive bacteria Bacillus cereus and Streptomyces chartreusis, a Gram-negative bacterium Escherichia coli and a yeast Candida albicans.

\section{Chemistry}

1-( $\beta$-D-glucopyranosyl)-indolin-2-one 6 was prepared in five steps via the corresponding indolic intermediate 3 which was synthesized by the indoline-indole method described by Mel'nik et al. [7]. Protection of the hydroxyl groups of the sugar moiety was performed with sodium hydride and benzyl bromide in the presence of tetrabutylammonium iodide [8]. Protected compound 4 was then brominated with pyridinium 


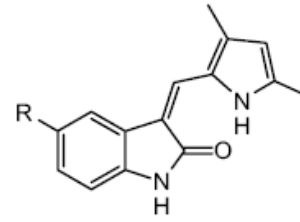

SU5416 R = H

VEGFR-2 inhibitor Phase III clinical trials

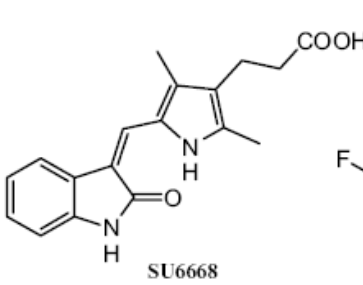

VEGRF-2, PDGFR- $\beta$, FGFR-1 inhibito Phase II clinical trials<smiles>CCN(CC)CCNC(=O)c1c(C)[nH]c(/C=C2\C(=O)Nc3ccc(F)cc32)c1C</smiles>

VEGFR-2 and PDGFR- $\beta$ inhibitor Phase I clinical trials

Fig. 1.

bromide perbromide and oxidized with peracetic acid leading to $3,3^{\prime}$-dibromo-1-(2,3,4,6-tetra- $O$-benzyl- $\beta$-D-glucopyrannosyl)-indolin-2-one 5 [9,10]. Finally, compound 6 was obtained by hydrogenolysis of the benzyl groups with concomitant elimination of the bromine atoms (Scheme 1).

Since in the previous described inhibitors of receptor tyrosine kinases, a conjugated aromatic system is attached in the 3 position of the oxindoles, compound 9, 3-benzylidenyl analogue of $\mathbf{6}$, was prepared in six steps via the indolic intermediate 4 (Scheme 2). The isatine derivative 7 was obtained by oxidation of $\mathbf{4}$ using chromium oxide [10]. Treatment of 7 with benzyltriphenylphosphonium bromide and butyllithium led to compound $\mathbf{8}$ as a mixture of $Z / E$ isomers. By analogy to the results obtained by Tacconi et al. [11] for the 3-benzylidenyl-indolin-2-one, $Z$ and $E$ vinylic protons were attributed, respectively, to the signals at 7.53 and $7.86 \mathrm{ppm}$. The $Z / E$ isomers ratio $(25 / 75)$ was determined from the ${ }^{1} \mathrm{H}$ NMR spectrum $\left(\mathrm{CDCl}_{3}\right)$ on the vinylic protons signals. These results were confirmed using quantum semiempirical calculations (Table 1). The comparison of the $E_{\text {a }}$ activation energy (energy difference between the reactants and the transition state) of $Z$ isomer and $E$ isomer for compound $\mathbf{8}$ showed that $E$ configuration is the major one. The value of $0.7 \mathrm{Kcal} \mathrm{mol}^{-1}$ of energy difference between $E_{\mathrm{a}}(Z)$ $E_{\mathrm{a}}(E)$ indicated that the theoretical $Z / E$ isomers ratio is equal to the experimental $Z / E$ isomers ratio [12].
Deprotection of the hydroxyl groups of the sugar moiety to give compound 9 was achieved using $N, N$-dimethylaniline and aluminium chloride [13].

To evaluate the influence of modifications on the heterocyclic moiety on the biological activity, especially the presence of a free $\mathrm{NH}$ in $\alpha$ position with respect to the carbonyl group, compound 12, isoster analogue of $\mathbf{6}$ with one nitrogen atom in position 3 was prepared from commercially available benzimidazolinone $\mathbf{1 0}$ (Scheme 3 ). Coupling with tetra- $O$ acetyl- $\alpha$-bromoglucose was performed with mercuric acetate in the presence of pyridine [14]. Compound $\mathbf{1 2}$ was obtained from 11 by deprotection of the hydroxyl groups of the sugar residue using potassium cyanide [15].

\section{Results and discussion}

\subsection{Kinases inhibition}

The in vitro kinase inhibitory activities were tested toward five kinases. Two structurally related split kinase domain RTKs (PDGFR- $\beta$, FGFR-1), endothelial cell specific receptor (KDR, Tie2) and EGFR. Compound 9 did not inhibit the kinases tested, it slightly activates the kinases. Only EGFR and Tie 2 were weakly inhibited by compound $\mathbf{6}$ and $\mathbf{1 2}$. Compared to the references used: staurosporine and SU5614 (an analogue of SU5416 (Fig. 1) with $\mathrm{R}=\mathrm{Cl}$ ), the results of

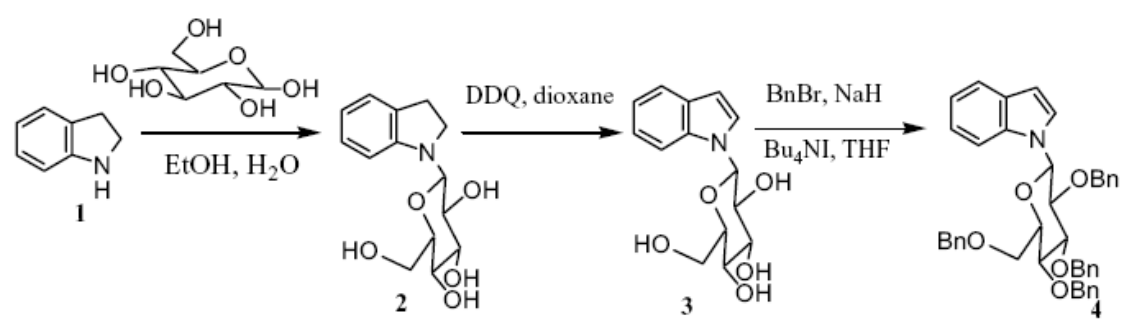

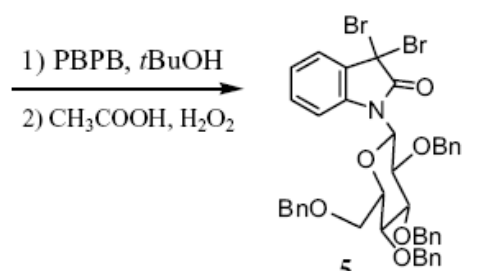<smiles>CCCCCCC(=O)OCC</smiles><smiles>O=C1Cc2ccccc2N1C1O[C@H](O)[C@H](O)[C@](O)(CO)C1O</smiles>

Scheme 1. 

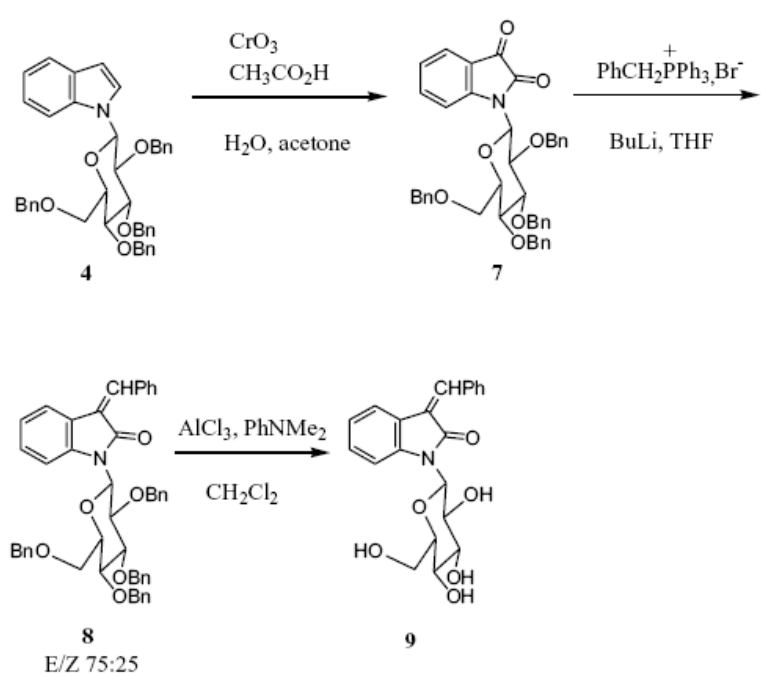

Scheme 2.

the inhibitory activities of these new compounds toward EGFR and Tie 2 were not very significative.

The potencies and selectivities toward various tyrosines kinases could be highly improved by chemical modifications on the aromatic moiety $[16,17]$. These chemical modifications are currently in progress in our laboratory with the aim of optimizing the biological activities of compounds $\mathbf{6}$ and 12.

Table 1

Computational results of isomers $E / Z$ ratio for compound $\mathbf{8}$

\begin{tabular}{llll}
\hline & $E$ isomer & $Z$ isomer \\
\hline$E_{\mathrm{a}}$ activational energy $\left(\mathrm{Kcal} \mathrm{mol}^{-1}\right)$ & 26.5 & & 27.2 \\
$E_{\mathrm{a}}(Z)-E_{\mathrm{a}}(E)\left(\mathrm{Kcal} \mathrm{mol}{ }^{-1}\right)$ & & 0.7 & \\
Theoretical ratio $E / Z$ & $75 \%$ & & $25 \%$ \\
Experimental ratio $E / Z$ & $75 \%$ & $25 \%$
\end{tabular}

The activation energy $E_{\mathrm{a}}$ is the energy difference between the reactants and the transition state.

\subsection{Antimicrobial properties}

The antimicrobial activities were tested against two Gram-positive bacteria (Bacillus cereus and Streptomyces chartreusis), a Gram-negative bacterium (Escherichia coli) and a yeast (Candida albicans) (Table 2). Compound 6 was inactive against the microorganisms tested. Compound $\mathbf{9}$, analogue of 6 with a benzylidenyl substituent at the 3 position was active against $B$. cereus. The most efficient was compound 12, an isoster of $\mathbf{6}$ with one nitrogen atom at the 3 position. This compound inhibits strongly the growth of the Gram-positive bacteria tested and that of E. coli. Moreover, compound $\mathbf{1 2}$ inhibits the growth of the yeast C. albicans.

In conclusion, a novel series of oxindoles and benzimidazolinones derivatives bearing a sugar residue on the aromatic nitrogen has been synthesized. Only the oxindole 6 and the benzimidazolinone $\mathbf{1 2}$ exhibited weak inhibitory activities against EGFR and Tie 2 kinases. However, compound $\mathbf{1 2}$ was strongly active against the bacteria tested. The biological targets of compound 12 remain to be determined. The pharmaceutical profile of this novel series could be optimized by structural modifications such as substitutions on the aromatic moiety and/or on the carbohydrate part. These modifications are currently in progress in our laboratory.

\section{Experimental}

\subsection{Chemistry}

IR spectra were recorded on a perkin-Elmer 881 spectrometer $\left(v\right.$ in $\left.\mathrm{cm}^{-1}\right)$. NMR spectra were performed on a Bruker AC $400\left({ }^{1} \mathrm{H}: 400 \mathrm{MHz},{ }^{13} \mathrm{C}: 100 \mathrm{MHz}\right.$ ) (chemical shifts $\delta$ in $\mathrm{ppm}$, the following abbreviations are used: singlet $(\mathrm{s})$, doublet $(\mathrm{d})$, triplet $(\mathrm{t})$, doubled triplet $(\mathrm{dt})$, doubled doublet (dd), doubled doubled doublet (ddd), multiplet (m),

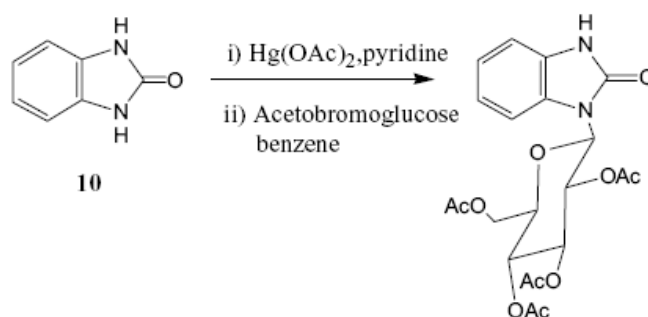

11

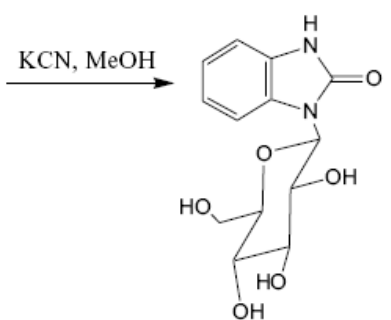

12

Scheme 3.

Table 2

Antimicrobial activities of compound 6,9,12 against two Gram-positive bacteria (Bacillus cereus and Streptomyces chartreusis), a Gram-negative bacterium (Escherichia coli) and a yeast (Candida albicans)

\begin{tabular}{lllll}
\hline Cpd & B. cereus ATCC 14579 & S. chartreusis NRRL 11407 & E. coli ATCC 11303 & C. albicans IP 444 \\
\hline $\mathbf{6}$ & - & - & - & - \\
$\mathbf{9}$ & + & nd & + & - \\
$\mathbf{1 2}$ & +++ & ++ & ++ & \pm \\
\hline
\end{tabular}

The size of the zones of growth inhibition was 13-16 mm (++++), 10-12 mm (+++), 8-9 (++), 7-8 mm (+), 6-7 mm ( \pm ); sp, inhibition of sporulation. 
broad signal (br s), tertiary carbons (C tert.), quaternary carbons (C quat.). Mass spectra (ES) were determined on a high resolution API Qstar Pulsar i at Organon (Riom, France). Chromatographic purifications were performed by flash silica gel Geduran SI 60 (Merck) $0.040-0.063 \mathrm{~mm}$ or Kieselgel 60 (Merck) $0.063-0.200 \mathrm{~mm}$ column chromatography. For purity tests, TLC were performed on fluorescent silica gel plates (60 F254 from Merck).

\subsubsection{1-( $\beta$-D-glucopyranosyl)-indoline (2)}

To a solution of indoline $\mathbf{1}(2 \mathrm{~g}, 16.8 \mathrm{mmol})$ in a mixture of ethanol $(120 \mathrm{ml})$ and water $(4 \mathrm{ml})$ was added D-glucose (1.41 g, $7.82 \mathrm{mmol})$. The resulting mixture was refluxed for $24 \mathrm{~h}$. Water was added after 7 and $14 \mathrm{~h}$ ( $1.6 \mathrm{ml}$ each time). The residue obtained after evaporation was purified by flash column chromatography (eluent EtOAc- $\mathrm{MeOH}$, from 98:2 to $90: 10)$ to give compound 2 ( $4.48 \mathrm{~g}, 16.0 \mathrm{mmol}, 95 \%$ yield) as a white solid: m.p. $113^{\circ} \mathrm{C}$. IR $(\mathrm{KBr}) v_{\mathrm{CO}} 1270 \mathrm{~cm}^{-1}, v_{\mathrm{C}=\mathrm{C}}$ $1610 \mathrm{~cm}^{-1}, v_{\mathrm{OH}} 3100-3700 \mathrm{~cm}^{-1}{ }^{1} \mathrm{H}$ NMR (400 MHz, DMSO- $\left.d_{6}\right): 2.91(\mathrm{~m}, 2 \mathrm{H}) ; 3.12(\mathrm{~m}, 1 \mathrm{H}) ; 3.21(\mathrm{~m}, 1 \mathrm{H}) ; 3.30$ $(\mathrm{m}, 2 \mathrm{H}) ; 3.38-3.54(\mathrm{~m}, 2 \mathrm{H}) ; 3.62(\mathrm{~m}, 2 \mathrm{H}) ; 4.35(\mathrm{t}, 1 \mathrm{H}$, $J=5.0 \mathrm{~Hz}, \mathrm{OH}) ; 4.64\left(\mathrm{~d}, 1 \mathrm{H}, J=8.0 \mathrm{~Hz}, \mathrm{H}_{1}\right) ; 4.91(\mathrm{~d}, 1 \mathrm{H}$, $J=5.0 \mathrm{~Hz}, \mathrm{OH}) ; 5.02($ br s, $2 \mathrm{H}, \mathrm{OH}) ; 6.59(\mathrm{t}, 2 \mathrm{H}, J=8.0 \mathrm{~Hz}$ ); $6.95(\mathrm{~d}, 1 \mathrm{H}, J=7.0 \mathrm{~Hz}) ; 7.00(\mathrm{~d}, 1 \mathrm{H}, J=8.0 \mathrm{~Hz}) .{ }^{13} \mathrm{C} \mathrm{NMR}$ $\left(100 \mathrm{MHz}, \mathrm{DMSO}-d_{6}\right): 28.3\left(\mathrm{CH}_{2}\right) ; 45.7\left(\mathrm{CH}_{2}\right) ; 61.5\left(\mathrm{C}_{6^{\prime}}\right)$; 70.7; 71.4; 78.4; 78.8; $85.7\left(\mathrm{C}_{1^{\prime}}, \mathrm{C}_{2^{\prime}}, \mathrm{C}_{3^{\prime}}, \mathrm{C}_{4^{\prime}}, \mathrm{C}_{5^{\prime}}\right)$; 108.1; $118.3 ; 125.0 ; 127.6\left(\mathrm{C}_{\text {tert }}\right) ; 130.2 ; 151.3\left(\mathrm{C}_{\text {quat }}\right)$.

\subsubsection{1-( $\beta$-D-glucopyranosyl)-indole (3)}

To a solution of $2(850 \mathrm{mg}, 3.02 \mathrm{mmol})$ in 1,4-dioxane $(170 \mathrm{ml})$ was added DDQ $(823 \mathrm{mg}, 3.62 \mathrm{mmol})$. The mixture was stirred at room temperature for $12 \mathrm{~h}$. After addition of saturated aqueous $\mathrm{NaHCO}_{3}$ and extraction with EtOAc, the organic phases were washed with saturated aqueous $\mathrm{NaCl}$ and dried over $\mathrm{MgSO}_{4}$. The solvent was removed and the residue purified by flash chromatography (eluent EtOAc$\mathrm{MeOH}, 98: 2)$ to give 3 (622 $\mathrm{mg}, 2.23 \mathrm{mmol}, 74 \%$ yield) as a salmon coloured solid: m.p. $90-95{ }^{\circ} \mathrm{C}$. IR $(\mathrm{KBr}) v_{\mathrm{C}=\mathrm{C}}$ $1610 \mathrm{~cm}^{-1}, v_{\mathrm{OH}} 3080-3700 \mathrm{~cm}^{-1 ;}{ }^{1} \mathrm{H}$ NMR (400 MHz, DMSO- $\left.d_{6}\right): 3.33(\mathrm{~m}, 1 \mathrm{H}) ; 3.45(\mathrm{~m}, 1 \mathrm{H}) ; 3.51(\mathrm{~m}, 2 \mathrm{H}) ; 3.75$ $(\mathrm{m}, 1 \mathrm{H}) ; 3.81(\mathrm{~m}, 1 \mathrm{H}) ; 4.59$ (br s, $1 \mathrm{H}, \mathrm{OH}) ; 5.15(\mathrm{~d}, 1 \mathrm{H}$, $J=5.0 \mathrm{~Hz}, \mathrm{OH}) ; 5.25(\mathrm{~d}, 2 \mathrm{H}, J=5.5 \mathrm{~Hz}, \mathrm{OH}) ; 5.46(\mathrm{~d}, 1 \mathrm{H}$, $\left.J=9.5 \mathrm{~Hz}, \mathrm{H}_{1^{\prime}}\right) ; 6.51(\mathrm{~d}, 1 \mathrm{H}, J=3.5 \mathrm{~Hz}) ; 7.09(\mathrm{t}, 1 \mathrm{H}$, $J=7.0 \mathrm{~Hz}) ; 7.18\left(\mathrm{dt}, 1 \mathrm{H}, J_{1}=7.9 \mathrm{~Hz} ; J_{2}=1.5 \mathrm{~Hz}\right) ; 7.56(\mathrm{~d}$, $1 \mathrm{H}, J=3.5 \mathrm{~Hz}) ; 7.59\left(\mathrm{dd}, 1 \mathrm{H}, J_{1}=8.0 \mathrm{~Hz} ; J_{2}=1.0 \mathrm{~Hz}\right) ; 7.61$ $(\mathrm{d}, 1 \mathrm{H}, J=8.0 \mathrm{~Hz}) .{ }^{13} \mathrm{C}$ NMR $\left(100 \mathrm{MHz}, \mathrm{DMSO}-d_{6}\right): 61.0$ $\left(\mathrm{C}_{6^{\prime}}\right)$; 69.9; 71.7; 77.6; 79.4; $84.8\left(\mathrm{C}_{1^{\prime}}, \mathrm{C}_{2^{\prime}}, \mathrm{C}_{3^{\prime}}, \mathrm{C}_{4^{\prime}}, \mathrm{C}_{5^{\prime}}\right)$; $101.6 ; 110.9 ; 119.7 ; 120.3 ; 121.2 ; 126.3\left(\mathrm{C}_{\text {tert }}\right) ; 128.5 ; 136.2$ ( $\left.\mathrm{C}_{\text {quat }}\right)$.

\subsubsection{1-(2,3,4,6-tetra-O-benzyl- $\beta$-D-glucopyranosyl $)$-indole (4)}

To a solution of $\mathbf{3}(300 \mathrm{mg}, 1.1 \mathrm{mmol})$ in dry THF $(15 \mathrm{ml})$ cooled to $0{ }^{\circ} \mathrm{C}$ was added $\mathrm{NaH}(420 \mathrm{mg}, 60 \%$ dispersion in mineral oil, $13.1 \mathrm{mmol}), \mathrm{Bu}_{4} \mathrm{NI}(31.2 \mathrm{mg}, 0.09 \mathrm{mmol})$, and benzyl bromide $(1.03 \mathrm{ml}, 12.9 \mathrm{mmol})$. The mixture was allowed to warm up to room temperature before refluxing for 6 h. Before evaporation, florisil (60/100mesh, Prolabo) was added to the reaction mixture to give a residue which was purified by flash column chromatography (eluent Cyclohexane-EtOAc 98:2) to give 4 ( $550 \mathrm{mg}, 0.86 \mathrm{mmol}$, $78 \%$ yield) as a yellow solid: m.p. $90{ }^{\circ} \mathrm{C}$. IR $(\mathrm{KBr}) v_{\mathrm{C}=\mathrm{C}} 1610 \mathrm{~cm}^{-1.1}{ }^{1} \mathrm{H}$ NMR (400 MHz, DMSO- $\left.d_{6}\right): 3.65(\mathrm{~d}, 1 \mathrm{H}, J=9.5 \mathrm{~Hz}) ; 3.75$ $(\mathrm{m}, 2 \mathrm{H}) ; 3.81(\mathrm{~d}, 1 \mathrm{H}, J=9.5 \mathrm{~Hz}) ; 3.95(\mathrm{~m}, 2 \mathrm{H}) ; 4.15(\mathrm{t}, 1 \mathrm{H}$, $J=9.5 \mathrm{~Hz}) ; 4.25(\mathrm{~d}, 1 \mathrm{H}, J=11.0 \mathrm{~Hz}) ; 4.50(\mathrm{~d}, 1 \mathrm{H}$, $J=12.5 \mathrm{~Hz}) ; 4.55(\mathrm{~d}, 1 \mathrm{H}, J=12.0 \mathrm{~Hz}) ; 4.65(\mathrm{~d}, 1 \mathrm{H}$, $J=11.0 \mathrm{~Hz}) ; 4.85(\mathrm{~d}, 1 \mathrm{H}, J=10.5 \mathrm{~Hz}) ; 4,90(\mathrm{br} \mathrm{s}, 2 \mathrm{H}) ; 5.82$ $\left(\mathrm{d}, 1 \mathrm{H}, J=9.0 \mathrm{~Hz}, \mathrm{H}_{1}\right) ; 6.65(\mathrm{~d}, 1 \mathrm{H}, J=3.0 \mathrm{~Hz}) ; 6.74(\mathrm{~d}, 2 \mathrm{H}$, $J=7.0 \mathrm{~Hz}) ; 7.09-7.39(\mathrm{~m}, 20 \mathrm{H}) ; 7.65(\mathrm{~d}, 1 \mathrm{H}, J=8.0 \mathrm{~Hz})$; $7.70(\mathrm{~d}, 1 \mathrm{H}, J=3.5 \mathrm{~Hz}) ; 7.74(\mathrm{~d}, 1 \mathrm{H}, J=8.0 \mathrm{~Hz}) .{ }^{13} \mathrm{C}$ NMR $\left(100 \mathrm{MHz}\right.$, DMSO- $\left.d_{6}\right): 68.7 ; 72.3 ; 73.3 ; 74.1 ; 74.6\left(\mathrm{C}_{6^{\prime}}+\right.$ $\mathrm{CH}_{2}$ benzyl $) ; 76.3 ; 77.6 ; 80.2 ; 83.9 ; 84.6\left(\mathrm{C}_{1^{\prime}}, \mathrm{C}_{2^{\prime}}, \mathrm{C}_{3^{\prime}}, \mathrm{C}_{4^{\prime}}\right.$, $\left.\mathrm{C}_{5^{\prime}}\right) ; 102.5 ; 111.1 ; 120.0 ; 120.6 ; 121.6 ; 126.5 ; 127.5-128.2$ $\left(\mathrm{C}_{\text {tert }}\right) ; 128.5 ; 135.9 ; 137.5 ; 138.1(2 \mathrm{C}) ; 138.5\left(\mathrm{C}_{\text {quat }}\right)$.

\subsubsection{3,3'-dibromo-1-(2,3,4,6-tetra-O-benzyl- $\beta$ - D-gluco- pyranosyl)-indolin-2-one (5)}

To a solution of indolic compound 4 (400 mg, $0.625 \mathrm{mmol})$ in $t$-butanol $(6.8 \mathrm{ml})$ was added pyridinium bromide perbromide (PBPB) $(858 \mathrm{mg}, 2.7 \mathrm{mmol})$. The reaction mixture was stirred at $40{ }^{\circ} \mathrm{C}$ for $24 \mathrm{~h}$ before evaporation and addition of a mixture EtOAc-water (1:1). After decantation, the aqueous phase was extracted twice with EtOAc. The organic phases were washed with saturated aqueous $\mathrm{NaCl}$ before drying over $\mathrm{MgSO}_{4}$ and concentrated under vacuum to give a crude residue $(512 \mathrm{mg}, 0.85 \mathrm{mmol}$ ) which was used directly without further purification for the next step. Acetic acid $(10.8 \mathrm{ml})$ then $\mathrm{H}_{2} \mathrm{O}_{2}(1.54 \mathrm{ml})$ were added. After stirring for $24 \mathrm{~h}$ at room temperature, a mixture of EtOAc-water (1:1) was added. After decantation, the aqueous layer was extracted twice with EtOAc. The organic phases were dried over $\mathrm{MgSO}_{4}$ and concentrated under vacuum to give a residue which was purified by flash chromatography (eluent Cyclohexane-EtOAc 90:10) to give 5 (355 mg, $0.437 \mathrm{mmol}$, $70 \%$ yield) as colorless solid: m.p. $50{ }^{\circ} \mathrm{C}$. IR $(\mathrm{KBr}) v_{\mathrm{C}=\mathrm{C}}$ $1610 \mathrm{~cm}^{-1}, v_{\mathrm{C}=\mathrm{O}} 1750 \mathrm{~cm}^{-1} .{ }^{1} \mathrm{H}$ NMR (300 MHz, DMSO$\left.d_{6}\right): 3.71(\mathrm{~s}, 2 \mathrm{H}) ; 3.85(\mathrm{~m}, 1 \mathrm{H}) ; 3.99(\mathrm{~m}, 2 \mathrm{H}) ; 4.21(\mathrm{br} \mathrm{s}, 1 \mathrm{H})$; $4.40(\mathrm{~m}, 1 \mathrm{H}) ; 4.54(\mathrm{~m}, 3 \mathrm{H}) ; 4.64(\mathrm{~d}, 1 \mathrm{H}, J=11.0 \mathrm{~Hz}) ; 4.81$ $(\mathrm{d}, 1 \mathrm{H}, J=11.0 \mathrm{~Hz}) ; 4.89$ (s, 2H); $5.66(\mathrm{~d}, 1 \mathrm{H}, J=9.0 \mathrm{~Hz}$, $\left.\mathrm{H}_{1^{\prime}}\right) ; 7.10(\mathrm{~m}, 2 \mathrm{H}) ; 7.17(\mathrm{~m}, 2 \mathrm{H}) ; 7.24(\mathrm{~m}, 2 \mathrm{H}) ; 7.26-7.40$ $(\mathrm{m}, 17 \mathrm{H}) ; 7.75(\mathrm{~d}, 1 \mathrm{H}, J=8.0 \mathrm{~Hz}) .{ }^{13} \mathrm{C}$ NMR $(100 \mathrm{MHz}$, DMSO- $\left.d_{6}\right): 68.3 ; 72.1 ; 73.7 ; 74.2 ; 74.6\left(\mathrm{C}_{6^{\prime}}+\mathrm{CH}_{2}\right.$ of benzyl groups); 76.1; 76.4; 77.3; 80.8; $84.4\left(\mathrm{C}_{1^{\prime}}, \mathrm{C}_{2^{\prime}}, \mathrm{C}_{3^{\prime}}, \mathrm{C}_{4^{\prime}}, \mathrm{C}_{5^{\prime}}\right)$; $124.6 ; 126.0 ; 127.4-128.2 ; 131.8\left(\mathrm{C}_{\text {tert }}\right) ; 130.1 ; 137.2 ; 138.0$; $138.2(2 \mathrm{C}) ; 138.4\left(\mathrm{C}_{\text {quat }}\right) ; 169.0(\mathrm{C}=\mathrm{O})$. The signal corresponding to $\mathrm{CBr}_{2}$ was not visible.

\subsubsection{1-( $\beta$-D-glucopyranosyl)-indolin-2-one (6)}

A mixture of $5(187 \mathrm{mg}, 0.59 \mathrm{mmol}), \mathrm{MeOH}(6 \mathrm{ml})$, EtOAc $(3 \mathrm{ml})$ and $10 \% \mathrm{Pd} / \mathrm{C}(40 \mathrm{mg})$ was hydrogenated at room temperature under 1 bar for $24 \mathrm{~h}$. After filtration over Celite and washing with $\mathrm{MeOH}$ and EtOAc, the filtrate was 
evaporated and the residue purified by flash chromatography (eluent EtOAc-MeOH, from 98:2 to 90:10) to give 6 (121 mg, $0.41 \mathrm{mmol}, 69 \%$ yield) as a white solid: m.p. $70-75{ }^{\circ} \mathrm{C}$. IR $(\mathrm{KBr}) v_{\mathrm{C}=\mathrm{C}} 1610 \mathrm{~cm}^{-1}, v_{\mathrm{C}=\mathrm{O}} 1705 \mathrm{~cm}^{-1}, v_{\mathrm{OH}}$ 3040-3680 $\mathrm{cm}^{-1}$; HRMS (ES) $[\mathrm{M}+\mathrm{Na}]^{+}$Calcd. for $\mathrm{C}_{14} \mathrm{H}_{17} \mathrm{NNaO}_{6}, 318.0948$; Found: 318.0950. ${ }^{1} \mathrm{H}$ NMR (400 MHz, DMSO- $\left.d_{6}\right)$ : 3.24-3.36 (m, 3H); $3.47(\mathrm{~m}, 1 \mathrm{H})$; $3.60(\mathrm{~s}, 2 \mathrm{H}) ; 3.75\left(\mathrm{dd}, 1 \mathrm{H}, J_{1}=11.5 \mathrm{~Hz}, J_{2}=5.5 \mathrm{~Hz}\right) ; 3.87$ $(\mathrm{m}, 1 \mathrm{H}) ; 4.62(\mathrm{t}, 1 \mathrm{H}, J=5.5 \mathrm{~Hz}, \mathrm{OH}) ; 5.10(\mathrm{~d}, 1 \mathrm{H}, J=5.0 \mathrm{~Hz}$, $\mathrm{OH}) ; 5.12(\mathrm{~d}, 1 \mathrm{H}, J=5.0 \mathrm{~Hz}, \mathrm{OH}) ; 5.20(\mathrm{~d}, 1 \mathrm{H}, J=9.0 \mathrm{~Hz}$, $\left.\mathrm{H}_{1^{\prime}}\right) ; 5.25(\mathrm{~d}, 1 \mathrm{H}, J=5.5 \mathrm{~Hz}, \mathrm{OH}) ; 7.05(\mathrm{t}, 1 \mathrm{H}, J=7.5 \mathrm{~Hz})$; $7.17(\mathrm{~d}, 1 \mathrm{H}, J=8.0 \mathrm{~Hz}) ; 7.25(\mathrm{t}, 1 \mathrm{H}, J=8.0 \mathrm{~Hz}) ; 7.30(\mathrm{~d}, 1 \mathrm{H}$, $J=7.5 \mathrm{~Hz}) .{ }^{13} \mathrm{C}$ NMR $\left(100 \mathrm{MHz}, \mathrm{CD}_{3} \mathrm{OD}\right): 37.0\left(\mathbf{C H}_{2} \mathrm{CO}\right)$; $63.1\left(\mathrm{C}_{6^{\prime}}\right) ; 70.7 ; 71.8 ; 79.3 ; 81.4 ; 83.8\left(\mathrm{C}_{1^{\prime}}, \mathrm{C}_{2^{\prime}}, \mathrm{C}_{3^{\prime}}, \mathrm{C}_{4^{\prime}}, \mathrm{C}_{5^{\prime}}\right)$; $113.2 ; 124.1 ; 125.8 ; 128.9\left(\mathrm{C}_{\text {tert }}\right) ; 126.3 ; 143.8\left(\mathrm{C}_{\text {quat }}\right) ; 178,0$ $(\mathrm{C}=\mathrm{O})$.

\subsubsection{1-(2,3,4,6-tetra-O-benzyl- $\beta$-D-glucopyranosyl $)$-indo- lin-2,3-dione (7)}

Chromium oxide (200 mg, $2.00 \mathrm{mmol}$ ) was added slowly to a solution of indole derivative $4(100 \mathrm{mg}, 0.156 \mathrm{mmol})$ in acetone $(0.5 \mathrm{ml})$, acetic acid $(1.7 \mathrm{ml}, 0.3 \mathrm{mmol})$ and water $(0.55 \mathrm{ml})$. The reaction mixture was stirred at room temperature for $2 \mathrm{~h}$. After addition of water and extraction with EtOAc, the organic phases were washed with water and saturated aqueous $\mathrm{NaCl}$ until neutral $\mathrm{pH}$. After drying over $\mathrm{MgSO}_{4}$, the solvent was removed and the residue purified by flash chromatography (eluent Cyclohexane-EtOAc, from $80: 20$ to $60: 40$ ) to give 7 (63 $\mathrm{mg}, 0.094 \mathrm{mmol}, 62 \%$ yield) as a yellow gum: IR $(\mathrm{NaCl}) v_{\mathrm{C}=\mathrm{C}} 1610 \mathrm{~cm}^{-1}, v_{\mathrm{C}=\mathrm{O}} 1740 \mathrm{~cm}^{-1}$; HRMS (ES) $[\mathrm{M}+\mathrm{Na}]^{+}$Calcd. for $\mathrm{C}_{42} \mathrm{H}_{39} \mathrm{NNaO}_{7}, 692.2618$; Found: $692.2633 ;{ }^{1} \mathrm{H}$ NMR (400 MHz, DMSO- $\left.d_{6}\right): 3.71(\mathrm{~d}$, $2 \mathrm{H}, J=3.0 \mathrm{~Hz}) ; 3.80(\mathrm{~m}, 1 \mathrm{H}) ; 3.89\left(\mathrm{dt}, 1 \mathrm{H}, J_{1}=9.5 \mathrm{~Hz}\right.$, $\left.J_{2}=3.5 \mathrm{~Hz}\right) ; 3.96(\mathrm{t}, 1 \mathrm{H}, J=8.5 \mathrm{~Hz}) ; 4.11(\mathrm{br} \mathrm{s}, 1 \mathrm{H}) ; 4.36(\mathrm{~d}$, $1 \mathrm{H}, J=12.0 \mathrm{~Hz}) ; 4.50(\mathrm{~d}, 2 \mathrm{H}, J=3.0 \mathrm{~Hz}) ; 4.64(\mathrm{~d}, 1 \mathrm{H}$, $J=11.0 \mathrm{~Hz}) ; 4.69(\mathrm{~d}, 1 \mathrm{H}, J=11.5 \mathrm{~Hz}) ; 4.81(\mathrm{~d}, 1 \mathrm{H}$, $J=11.0 \mathrm{~Hz}) ; 4.90(\mathrm{~s}, 2 \mathrm{H}) ; 5.54\left(\mathrm{~d}, 1 \mathrm{H}, J=9.0 \mathrm{~Hz}, \mathrm{H}_{1^{\prime}}\right) ; 6.95$ $(\mathrm{d}, 2 \mathrm{H}, J=7.0 \mathrm{~Hz}) ; 7.04-7.13(\mathrm{~m}, 3 \mathrm{H}) ; 7.20(\mathrm{t}, 1 \mathrm{H}$, $J=8.0 \mathrm{~Hz}) ; 7.25\left(\mathrm{dd}, 1 \mathrm{H}, J_{1}=7.5 \mathrm{~Hz}, J_{2}=2.5 \mathrm{~Hz}\right) ; 729-7.40$ $(\mathrm{m}, 15 \mathrm{H}) ; 7.55(\mathrm{~d}, 1 \mathrm{H}, J=7.5 \mathrm{~Hz}) ; 7.60(\mathrm{t}, 1 \mathrm{H}, J=8.0 \mathrm{~Hz})$. ${ }^{13} \mathrm{C} \mathrm{NMR}\left(100 \mathrm{MHz}\right.$, DMSO- $d_{6}$ ): 68.4; 72.2; 73.9; 74.2; 74.7 $\left(\mathrm{C}_{6^{\prime}}+\mathrm{CH}_{2}\right.$ of the benzyl groups); 76.3; 76.5; 77.3; 80.0; 84.9 $\left(\mathrm{C}_{1^{\prime}}, \mathrm{C}_{2^{\prime}}, \mathrm{C}_{3^{\prime}}, \mathrm{C}_{4^{\prime}}, \mathrm{C}_{5^{\prime}}\right) ; 117.5 ; 137.6 ; 138.0 ; 138.1$ (2C); $138.4\left(\mathrm{C}_{\text {quat }}\right) ; 123.7 ; 124.8 ; 127.4-128.3 ; 138.2\left(\mathrm{C}_{\text {tert }}\right)$; $157.4 ; 182.0(\mathrm{C}=\mathrm{O})$.

4.1.7. 3-benzylidenyl-1-(2,3,4,6-tetra-O-benzyl- $\beta$-D-glucopyranosyl)-indolin-2-one ( 8 )

A solution of butyllithium ( $2 \mathrm{M}$ in cyclohexane, $0.11 \mathrm{ml}$ ) was added to a suspension of benzyltriphenylphosphonium bromide $(92 \mathrm{mg}, 0.21 \mathrm{mmol})$ in THF $(5 \mathrm{ml})$. This mixture was stirred at room temperature for $1 \mathrm{~h}$ before addition of a solution of 7 (100 mg, $0.15 \mathrm{mmol})$ in THF $(5 \mathrm{ml})$. Saturated aqueous $\mathrm{NaCl}$ was added after stirring at room temperature for $1 \mathrm{~h}$. The aqueous layer was extracted with EtOAc. The combined organic phases were dried over $\mathrm{MgSO}_{4}$, evapo- rated and the residue was purified by chromatography (eluent Toluene-THF 98:2) to give a mixture of E/Z 8 (109 mg, $0.147 \mathrm{mmol}, 98 \%$ yield) as a yellow gum: IR $(\mathrm{NaCl}) v_{\mathrm{C}=\mathrm{C}}$ $1610,1640 \mathrm{~cm}^{-1},{ }^{{ } \mathrm{C}=\mathrm{O}} 1720 \mathrm{~cm}^{-1 ;}{ }^{1} \mathrm{H}$ NMR $(400 \mathrm{MHz}$, $\mathrm{CDCl}_{3}$ ) for the major $E$ isomer: $3.72(\mathrm{~m}, 3 \mathrm{H}) ; 3.88(\mathrm{~m}, 3 \mathrm{H})$; $4.33(\mathrm{~d}, 1 \mathrm{H}, J=11.0 \mathrm{~Hz}) ; 4.49(\mathrm{~d}, 1 \mathrm{H}, J=12.0 \mathrm{~Hz}) ; 4.59(\mathrm{~m}$, $2 \mathrm{H}) ; 4.68(\mathrm{~d}, 1 \mathrm{H}, J=11.0 \mathrm{~Hz}) ; 4.91(\mathrm{~m}, 3 \mathrm{H}) ; 5.68(\mathrm{~d}, 1 \mathrm{H}$, $\left.J=9.0 \mathrm{~Hz}, \mathrm{H}_{1^{\prime}}\right) ; 6.85\left(\mathrm{td}, 1 \mathrm{H}, J_{1}=7.5 \mathrm{~Hz}, J_{2}=1.0 \mathrm{~Hz}\right) ; 6.96$ $(\mathrm{m}, 1 \mathrm{H}) ; 7.02(\mathrm{~m}, 2 \mathrm{H}) ; 7.09(\mathrm{~m}, 2 \mathrm{H}) ; 7.15(\mathrm{t}, 1 \mathrm{H}, J=7.5 \mathrm{~Hz})$; $7.22-7.33(\mathrm{~m}, 17 \mathrm{H}) ; 7.46(\mathrm{~m}, 3 \mathrm{H}) ; 7.61(\mathrm{~d}, 1 \mathrm{H}, J=7.6 \mathrm{~Hz})$; $7.64(\mathrm{~d}, 1 \mathrm{H}, J=6.7 \mathrm{~Hz}) ; 7.86(\mathrm{~s}, 1 \mathrm{H}, \mathrm{CHPh}) .{ }^{13} \mathrm{C} \mathrm{NMR}$ $\left(100 \mathrm{MHz}\right.$, DMSO- $\left.d_{6}\right): 69.9 ; 73.5 ; 75.1 ; 75.5 ; 75.9\left(\mathrm{C}_{6^{\prime}}+\right.$ $\mathrm{CH}_{2}$ of the benzyl groups); 77.7; 78.0; 78.8; 81.4; 86.1; $\left(\mathrm{C}_{1^{\prime}}\right.$, $\left.\mathrm{C}_{2^{\prime}}, \mathrm{C}_{3^{\prime}}, \mathrm{C}_{4^{\prime}}, \mathrm{C}_{5^{\prime}}\right) ; 121.7 ; 127.4 ; 135.5 ; 139.4$ (2C); 139.5; $139.8(2 \mathrm{C})\left(\mathrm{C}_{\text {quat }}\right) ; 123.5 ; 123.6 ; 128.7-129.5 ; 130.1 ; 130.6$; $131.3 ; 133.2 ; 138.7 ; 139.6\left(\mathrm{C}_{\text {tert }}\right) ; 168.4(\mathrm{C}=\mathrm{O})$.

\subsubsection{3-benzylidenyl-1- $\beta$-D-glucopyranosyl-indolin-2-one (9)}

To a solution of $\mathbf{8}(152 \mathrm{mg}, 0.2 \mathrm{mmol})$ in $\mathrm{CH}_{2} \mathrm{Cl}_{2}(0.5 \mathrm{ml})$, dimethylaniline $(78 \mu \mathrm{l}, 0.6 \mathrm{mmol})$ and $\mathrm{AlCl}_{3}(109 \mathrm{mg}$, $0.8 \mathrm{mmol}$ ) were added. The reaction mixture was stirred at room temperature for $21 \mathrm{~h}$. Dimethylaniline and $\mathrm{AlCl}_{3}$ were added after 1 and $2 \mathrm{~h}(0.1 \mathrm{ml}$ and $100 \mathrm{mg}$ respectively each time). $\mathrm{HCl} 2 \mathrm{M}(1.0 \mathrm{ml})$ was added to the reaction mixture before extraction with EtOAc. The organic phase was washed successively with $5 \%$ aqueous $\mathrm{NaHCO}_{3}$, saturated aqueous $\mathrm{NaCl}$ and water. After drying over $\mathrm{MgSO}_{4}$, the solvent was removed and the residue purified by chromatography (eluent EtOAc-MeOH, from 2:98 to 10:90) to give a mixture of $E / Z 9$ (20 mg, $0.05 \mathrm{mmol}, 26 \%$ yield) as an orange-brown amorphous solid: IR $(\mathrm{KBr}) v_{\mathrm{C}=\mathrm{C}} 1610 \mathrm{~cm}^{-1}$, ${ }^{v_{\mathrm{C}=\mathrm{O}}} 1705 \mathrm{~cm}^{-1}, v_{\mathrm{OH}} 3040-3680 \mathrm{~cm}^{-1}$. HRMS (ES) [M + $\mathrm{Na}]^{+}$Calcd. for $\mathrm{C}_{21} \mathrm{H}_{21} \mathrm{NNaO}_{6}, 406.1261$; Found: 406.1259 . ${ }^{1} \mathrm{H}$ NMR (400 MHz, DMSO- $d_{6}$ ) for the major $E$ isomer: 3.30 $(\mathrm{m}, 1 \mathrm{H}) ; 3.37(\mathrm{~m}, 2 \mathrm{H}) ; 3.52(\mathrm{~m}, 1 \mathrm{H}) ; 3.77(\mathrm{~m}, 1 \mathrm{H}) ; 3.91(\mathrm{~m}$, $1 \mathrm{H}) ; 4.65$ (br s, $1 \mathrm{H}, \mathrm{OH}) ; 5.65$ (br s, $1 \mathrm{H}, \mathrm{OH}) ; 5.73$ (br s, $1 \mathrm{H}$, $\mathrm{OH}) ; 5.31$ (br s, $1 \mathrm{H}, \mathrm{OH}) ; 5.32\left(\mathrm{~d}, 1 \mathrm{H}, J=8.5 \mathrm{~Hz}, \mathrm{H}_{1^{\prime}}\right) ; 6.93$ $\left(\mathrm{t}, 1 \mathrm{H}, J=8.0, \mathrm{H}_{\text {arom }}\right)$ ) $7.25(\mathrm{~d}, 1 \mathrm{H}, J=7.5 \mathrm{~Hz}) ; 7.34(\mathrm{~m}, 2 \mathrm{H})$; 7.50-7.64 (m, 3H); $7.75(\mathrm{~m}, 2 \mathrm{H}) ; 7.80$ (s, 1H, CHPh).

\subsubsection{1-(2,3,4,6-tetra-O-acetyl- $\beta$-D-glucopyranosyl)-benzi- midazolin-2-one (11) \\ Compound 11 was obtained as a white solid according to} the procedure described by Zinner and Peseke [14]: m.p. $248{ }^{\circ} \mathrm{C}$ (m.p.litt $\left.=244-246{ }^{\circ} \mathrm{C}\right)$ [14], ${ }^{1} \mathrm{H}$ NMR (400 MHz, DMSO- $\left.d_{6}\right): 1.76 ; 1.90 ; 1.97 ; 1.99\left(4 \mathrm{~s}, 12 \mathrm{H}, \mathrm{CH}_{3}\right.$ acetate); $4.08(\mathrm{~d}, 2 \mathrm{H}, J=3.0 \mathrm{~Hz}) ; 4.28\left(\mathrm{dt}, 1 \mathrm{H}, J_{1}=10.0 \mathrm{~Hz}\right.$; $\left.J_{2}=3.5 \mathrm{~Hz}\right) ; 5.24(\mathrm{t}, 1 \mathrm{H}, J=9.5 \mathrm{~Hz}) ; 5.53(\mathrm{t}, 1 \mathrm{H}, J=9.5 \mathrm{~Hz})$; $5.57(\mathrm{t}, 1 \mathrm{H}, J=9.5 \mathrm{~Hz}) ; 5.88\left(\mathrm{~d}, 1 \mathrm{H}, J=9.0 \mathrm{~Hz}, \mathrm{H}_{1^{\prime}}\right) ; 6.96(\mathrm{~m}$, $3 \mathrm{H}) ; 7.42\left(\mathrm{dd}, 1 \mathrm{H}, J_{1}=6.0 \mathrm{~Hz} ; J_{2}=2.5 \mathrm{~Hz}\right) ; 11.0(\mathrm{~s}, 1 \mathrm{H}, \mathrm{NH})$.

\subsubsection{1-( $\beta$-D-glucopyranosyl)-benzimidazolin-2-one (12)}

Potassium cyanide $(58.6 \mathrm{mg}, 0.9 \mathrm{mmol})$ was added to a solution of compound $11(500 \mathrm{mg}, 0.9 \mathrm{mmol})$ in methanol $(10 \mathrm{ml})$. The mixture was stirred at room temperature for 
$24 \mathrm{~h}$. Evaporation of the reaction mixture gave a residue which was purified by flash chromatography (eluent EtOAc$\mathrm{MeOH}$, from 95:5 to 90:10) to give 12 (154 mg, $0.50 \mathrm{mmol}$, $56 \%$ yield) as a white solid; m.p. $150{ }^{\circ} \mathrm{C}$; IR $(\mathrm{KBr}) v_{\mathrm{C}=\mathrm{C}}$ $1625 \mathrm{~cm}^{-1}, v_{\mathrm{C}=\mathrm{O}} 1700 \mathrm{~cm}^{-1}, v_{\mathrm{OH}} 3000-3600 \mathrm{~cm}^{-1}$. HRMS (ES) $[\mathrm{M}+\mathrm{Na}]^{+}$: Calcd. for $\mathrm{C}_{13} \mathrm{H}_{16} \mathrm{~N}_{2} \mathrm{O}_{6}, 319.0900$, Found: 319.0906. ${ }^{1} \mathrm{H}$ NMR (300 MHz, DMSO- $\left.d_{6}\right): 3.29-3.40$ (m, $3 \mathrm{H}) ; 3.57\left(\mathrm{dt}, 1 \mathrm{H}, J_{1}=11.5 \mathrm{~Hz} ; J_{2}=6.0 \mathrm{~Hz}\right) ; 3.86(\mathrm{dd}, 1 \mathrm{H}$, $\left.J_{1}=11.5 \mathrm{~Hz} ; J_{2}=6.0 \mathrm{~Hz}\right) ; 4.01(\mathrm{~m}, 1 \mathrm{H}) ; 4.69(\mathrm{t}, 1 \mathrm{H}$, $J=5.5 \mathrm{~Hz}, \mathrm{OH}) ; 5.19(\mathrm{~d}, 1 \mathrm{H}, J=5.0 \mathrm{~Hz}, \mathrm{OH}) ; 5.21(\mathrm{~d}, 1 \mathrm{H}$, $J=5.0 \mathrm{~Hz}, \mathrm{OH}) ; 5.29\left(\mathrm{~d}, 1 \mathrm{H}, J=9.5 \mathrm{~Hz}, \mathrm{H}_{1^{\prime}}\right.$ ) $; 5.31(\mathrm{~d}, 1 \mathrm{H}$, $J=5.5 \mathrm{~Hz}, \mathrm{OH}) ; 7.11(\mathrm{~m}, 3 \mathrm{H}) ; 7.29\left(\mathrm{dd}, 1 \mathrm{H}, J_{1}=8.0 \mathrm{~Hz}\right.$; $\left.J_{2}=3.0 \mathrm{~Hz}\right) ; 11.0(\mathrm{~s}, 1 \mathrm{H}, \mathrm{NH}) .{ }^{13} \mathrm{C} \mathrm{NMR}(100 \mathrm{MHz}$, $\left.\mathrm{CD}_{3} \mathrm{OD}\right): 63.1\left(\mathrm{C}_{6^{\prime}}\right) ; 71.5 ; 71.8 ; 79.4 ; 81.4 ; 84.7\left(\mathrm{C}_{1^{\prime}}, \mathrm{C}_{2^{\prime}}\right.$, $\left.\mathrm{C}_{3^{\prime}}, \mathrm{C}_{4^{\prime}}, \mathrm{C}_{5^{\prime}}\right) ; 110.8 ; 112.2 ; 122.6 ; 123.4\left(\mathrm{C}_{\text {tert }}\right) ; 130.1(2 \mathrm{C})$ $\left(\mathrm{C}_{\text {quat }}\right) ; 156.8(\mathrm{C}=\mathrm{O})$.

\subsection{Computational methods}

Quantum semi-empirical calculations SAM1 [18] were carried out using AMPAC 7.0 [19] program. The FULLCHN (unpublished results) procedure was used to locate the transition state. From this transition state geometry, the Intrinsic Reaction Coordinate $[20,21]$ (IRC) calculation was performed to generate the correct reactants and products states by following the reaction coordinate in both directions leading to reactants and products.

\subsection{Kinases inhibition}

In order to determine the in vitro activity of compounds against different protein kinases, biochemical assays were performed using glutathione $S$-transferase-fusion proteins containing the complete cytoplasmic domain of the Receptor Tyrosine Kinase (GST-RTK cyt). Activities were determined at $10 \mu \mathrm{M}$ in a flashplate assay. Phosphorylation inhibition was calculated as follows: $\%$ of inhibition $=\left[\mathrm{cpm}_{(- \text {molecule })}-\right.$ $\left.\mathrm{cpm}_{(+ \text {molecule })} / \mathrm{cpm}_{(- \text {molecule })}\right] \times 100$ SU5614 (Calbiochem cat Nos. 572 632) at $1 \mu \mathrm{M}$ was used as the reference inhibitor for KDR and PDGFR, and Staurosporine (Sigma S4400) at $0.1 \mu \mathrm{M}$ for the other kinases.

Compounds ( $1 \mu \mathrm{M}, 0.1 \%$ DMSO final) were incubated during $5 \mathrm{~min}$ with GST-RTK cyt $(200 \mathrm{ng})$ in a Phosphatebuffer (20 mM MOPS, $10 \mathrm{mM} \mathrm{MgCl} 2,10 \mathrm{mM} \mathrm{MnCl}_{2}, 1 \mathrm{mM}$ DTT, $2.5 \mathrm{mM}$ EGTA, $10 \mathrm{mM} \beta$-Glycerophosphate, $1 \mathrm{mM}$ $\mathrm{Na}_{3} \mathrm{VO}_{4}, 1 \mathrm{mM} \mathrm{NaF}$ ) in flashplate (NEN). The reaction was started by addition of GST-PLC- $\gamma$ as the substrate $(2 \mu \mathrm{g})$, $2 \mu \mathrm{Ci} \gamma^{33} \mathrm{P}$-ATP, in presence of $2 \mu \mathrm{M}$ cold ATP. Reaction was performed during $1 \mathrm{~h}$ at $37^{\circ} \mathrm{C}$, stopped by addition of EDTA (100 $\mathrm{mM}$ final), washed with PBS-tween $0.1 \%$ and the flashplate was counted using a micro $\beta$ Wallac apparatus.

\subsection{Antibiogram tests}

Four strains were tested, two Gram-positive bacteria (B. cereus ATCC 14579, S. chartreusis NRRL 11407), a
Gram-negative bacterium (E. coli ATCC 11303) and a yeast (C. albicans 444 from the Pasteur Institute, Paris). The antimicrobial activity was determined by the conventional paper disk (Durieux No. 268; $6 \mathrm{~mm}$ in diameter) diffusion method using the following nutrient media: Mueller-Hinton (Difco) for B. cereus and E. coli, Sabouraud agar (Difco) for C. albicans and Emerson agar $(0.4 \%$ beef extract, $0.4 \%$ peptone, $1 \%$ dextrose, $0.25 \% \mathrm{NaCl}, 2 \%$ agar, $\mathrm{pH} 7.0$ ) for the Streptomyces strains. Paper disks impregnated with solutions in DMSO (300 $\mu \mathrm{g}$ of drug per disk) were placed on Petri dishes. Growth inhibition was examined after $24 \mathrm{~h}$ incubation at $27^{\circ} \mathrm{C}$.

\section{Acknowledgements}

The authors are grateful to Christel Bufferne and Françoise Lombard (Organon, Riom) for the HRMS (ES) analyses.

\section{References}

[1] P. Haluska, A.A. Adjei, Curr. Opin. Investig. Drugs 2 (2001) 280-286.

[2] L.M. Toledo, N.B. Lydon, D. Elbaum, Curr. Med. Chem. 6 (1999) $775-805$.

[3] A.D. Laird, J.M. Cherrington, Expert Opin. Investig. Drugs 12 (2003) $51-64$.

[4] L. Sun, C. Liang, S. Shirazian, Y. Zhou, T. Miller, J. Cui, J.Y. Fukada, J.Y. Chu, A. Nematalla, X. Wang, H. Chen, A. Sistla, T.C. Luu, F. Tang, J. Wei, C. Tang, J. Med. Chem. 46 (2003) 1116-1119.

[5] S.K. Sridhar, M. Saravanan, A. Ramesh, Eur. J. Med. Chem. 36 (2001) 615-625.

[6] M.N.S. Saudi, M.M.A. Semary, G. Sawaf, Pharmazie 57 (2002) 519522.

[7] S. Ya. Mel'nik, A.A. Bakhmedova, L.D. Garaeva, O.V. Goryunova, T.D. Miniker, I.L. Plikhtyak, L.V. Ektova, T.P. Ivanova, I.V. Yartseva, Bioorganicheskya Khimiya 22 (1996) 467-476.

[8] S. Czernecki, C. Georgoulis, C. Provelenghiou, Tetrahedron Lett. 17 (1976) 3535-3536.

[9] A. Da Settimo, C. Menicagli, E. Nannipieri, J. Org. Chem. 39 (1974) 1995-1998.

[10] A. Marfat, M.P. Carta, Tetrahedron Lett. 28 (1987) 4027-4030.

[11] A.C. Coda, A.G. Invernizzi, P.P. Righetti, G. Tacconi, G. Gatti, J. Chem. Soc. Perkin Trans. II (1984) 615-619.

[12] E.L. Eliel, Stereochemistry of Carbon Compounds, Mac Graw Hill, 1962, pp. 207.

[13] T. Akiyama, H. Hirofuji, S. Ozaki, Tetrahedron Lett. 32 (1991) 13211324.

[14] V.H. Zinner, K. Peseke, J. Prakt. Chem. 312 (1970) 307-313.

[15] J. Herzig, J. Org. Chem. 51 (1986) 727-730.

[16] P.C. Tang, L. Sun, G. McMahon, US Patent (1999) US 5886020 , Chem. Abstr. 130, 252240n.

[17] K.P. Hirth, G. McMahon, L.K. Shawver, WO Patent (1997) WO 97/34920, Chem. Abstr. 127, 314804.

[18] M.J.S. Dewar, J. Caoxian, Y. Jianguo, Tetrahedron 49 (1993) 5003 5038.

[19] Ampac7-0, 2000 Semichem, 7128 Summit, Shawnee, KS 66216.

[20] C. Gonzalez, H.B. Schlegel, J. Chem. Phys. 90 (1989) 2154-2161.

[21] C. Gonzalez, H.B. Schlegel, J. Phys. Chem. 94 (1990) 5523-5527. 